\title{
What Conservation Biologists Can Do to Counter Trap-Neuter-Return: Response to Longcore et al.
}

\author{
CHRISTOPHER A. LEPCZYK,${ }^{*}$ NICO DAUPHINÉ,$\dagger$ DAVID M. BIRD,$\ddagger$ SHEILA CONANT,$\S$ \\ ROBERT J. COOPER, ${ }^{* *}$ DAVID C. DUFFY,$\dagger+$ PAMELA JO HATLEY, $\S \S$ PETER P. MARRA, ${ }^{* * *}$ \\ ELIZABETH STONE, $\nmid \dagger \dagger$ AND STANLEY A. TEMPLE $\ddagger £ \S \S$
}

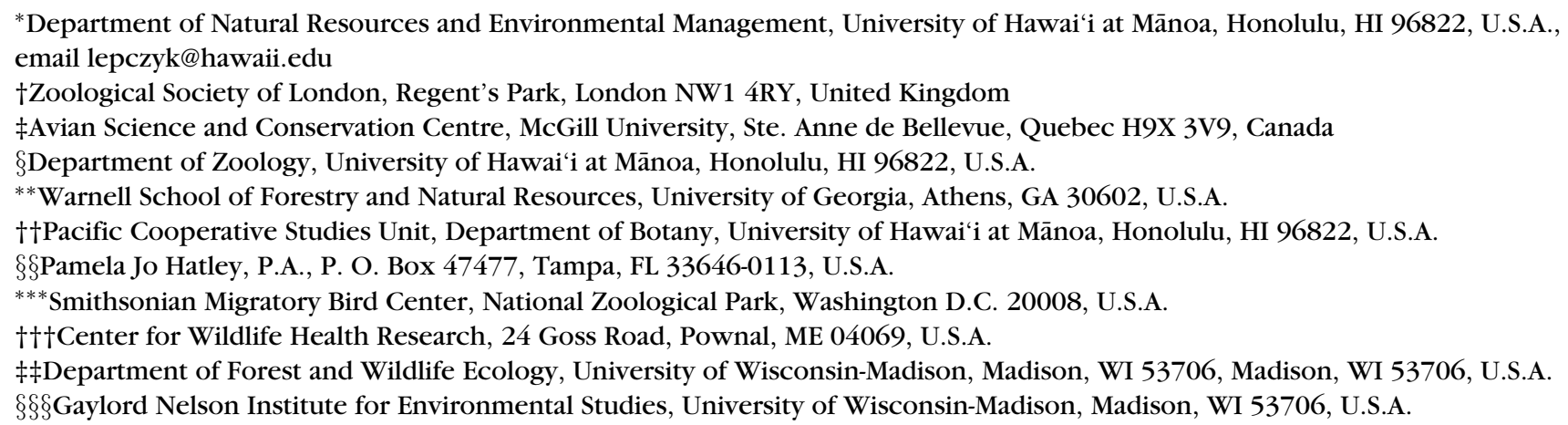

We applaud the recent essay by Longcore et al. (2009) in raising the awareness about trap-neuter-return (TNR) to the conservation community. Trap-neuter-return has gained a great deal of traction in recent years as an accepted form of feral cat management, but as noted by the authors, it has not lived up to the ideal it proffers. We concur with the authors' thoughts and findings on TNR and want to make several additional points about feral cat management and TNR regarding environmental conservation.

First, TNR is often presented to policy makers and the public as a scientifically valid and humane way of controlling and managing homeless and unwanted cats. Proponents of TNR are well organized and push for TNRfriendly policies in communities and shelters around the United States, often with little opposition from the conservation biology and wildlife ecology communities. The reasons behind this lack of opposition are unclear, but it may be that conservation biologists and wildlife ecologists believe the issue of feral cats has already been studied enough and that the work speaks for itself, suggesting that no further research is needed. Or, they simply do not want to devote time and energy to the issue and are unaware of policy actions.
Regardless of why the scientific and management communities have remained relatively silent, it is imperative that we now begin speaking out (sensu Nelson \& Vucetich 2009). By way of example, those of us who are conservation biologists should look to the evolutionary biology community. When local policies or regulations are put forth that promote the teaching of creationism or intelligent design, the evolutionary biologists have responded in force from across the nation and world. Such responses have been successful in defeating attempts to favor the teaching of creationism or intelligent design and serve to remind the public that the scientific evidence overwhelmingly supports the theory of evolution. We the conservation community should consider the issue of TNR in the same light and challenge such propositions when they are raised. Without such challenges by those who are knowledgeable about the subject, we simply allow the use of TNR to grow and thereby gain further acceptance.

Second, the conservation biology and wildlife ecology communities need to build bridges with the animal welfare, veterinary, and public-health communities. Many organizations within these communities oppose TNR, cat colonies, and outdoor cats in general, because cats living 


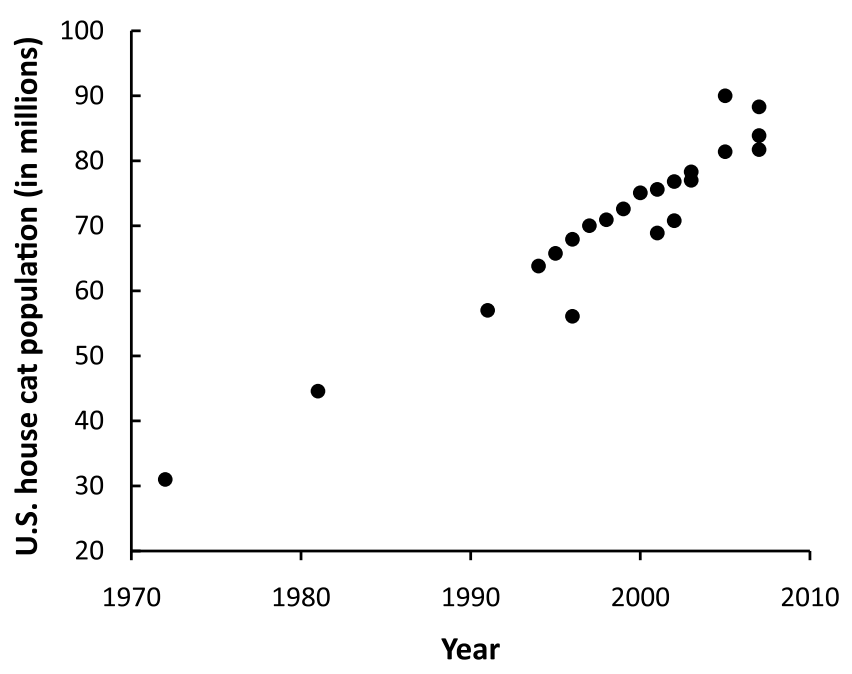

Figure 1. Growth of the bouse cat population in the United States over time. Population estimates were derived from manufacturers of pet food, American Pet Products Manufacturers Association, American Veterinary Medical Association, and similar organizations.

in these conditions tend to have shorter lives, higher levels of parasites and disease, and generally poorer health. In fact, across the United States cats are the leading rabies vector among domestic animals (Blanton et al. 2007). Although proponents of TNR assert that they are providing services that allow cats to live full and healthy lives, freeroaming and feral cats are often in very poor condition (Jessup 2004). The animal welfare community opposes "cat hoarding," whereby people care for more pets than they can adequately support, because it is considered inhumane. Trap-neuter-return is essentially cat hoarding without walls. Considering that most communities have laws banning animal hoarding, we should consider the same standard for outdoor cats as those that are in a person's home.

What then to do about the problems of outdoor cats, cat colonies, and unwanted cats? The population of owned cats is growing (Fig. 1) and likely mirrored by a growing population of feral cats (Schmidt et al. 2007). This problem almost certainly has been exacerbated by the current economic recession, whereby increasing numbers of pets are being abandoned or surrendered to shelters (American Society for the Prevention of Cruelty to Animals 2009). Concurrently, there are increasing efforts to convert animal shelters into "no kill" facilities. As a result, we face the conundrum of how to humanely deal with more and more feral cats. There is no simple answer that will please all parties, in large part because we now find ourselves in the unpleasant position of having far too many feral cats in the environment with no single solution that is acceptable to all. Ultimately, the issue of feral cats is a social problem and one that many find uncomfortable due to the highly charged emotions asso- ciated with it. No one wants to euthanize animals and no one likes to see them suffer. But unfortunately, the consequences of allowing cats to roam wild or live in colonies with extremely poor health conditions are less humane than euthanasia. We as a society do not allow dogs, ferrets, livestock, or other domestic animals to roam free or live in the wild in colonies and the same should be true for cats. Cats deserve the same humane treatment and legal regards as dogs and other pets. Likewise, wild animals (i.e., amphibians, birds, mammals, and reptiles) deserve humane treatment and should not be reduced to second-class treatment at the expense of cats (or other pets).

We suggest that the following actions should be encouraged. First, conservation biologists, wildlife ecologists, and the like should have open dialogues with the animal welfare, sheltering, veterinary, and public-health communities. These communities generally agree on a desire to promote animal welfare and reduce cat overpopulation. If we do not begin to work together, we will face strong obstacles in reducing the numbers of feral cats present on our landscapes.

Second, the wildlife and conservation communities need to challenge policies that are put forth to allow or promote feral cat colonies and TNR. Conservation biologists have just as much opportunity to make their points at local meetings, through the news media, and at outreach events as do TNR proponents. Although feral cats may not be the issue we are most interested in or want to pursue with our time, we should remind ourselves of our responsibility to provide scientific information to the public with regard to wildlife and the environment.

Third, the wildlife and conservation communities should advocate for policies that encourage responsible pet ownership as well as for enforcement of existing policies. This includes requiring licenses for cats, substantially decreasing unwanted breeding of pet cats through mandatory or subsidized spaying and neutering, and requiring cats to be kept under their owners' control at all times when outdoors. In many cases, local ordinances requiring owners to keep their domestic animals under control are enforced for dogs, but not for cats. In these cases, such policies are already in place but require increased enforcement in order to be effective.

Fourth, "releasing cats into the wild and supporting feral cat colonies is a violation of the Migratory Bird Treaty Act and the Endangered Species Act, as well as laws prohibiting animal abandonment (Winter 2003)." Thus, it may become incumbent upon us to take legal action against colonies and colony managers, particularly in areas that provide habitat for migratory birds or endangered species.

Fifth, we should seek laws making it illegal to maintain cat colonies on public lands. Sixth, we need to increase public awareness about being a responsible pet owner, not just for the benefit of cats (and other domestic animals 
as well), but also for individuals of wildlife species and the environment.

Finally, we need to move away from the prevailing view that depredation of individual wildlife species does not matter as long as their populations are intact. If we are to consider cats from an individualistic viewpoint, then the same argument must be made for wild animals. The issue of feral cats is not going away any time soon, and no matter what options are taken, it may well be a generation or more before we can expect broad-scale changes in human behavior regarding outdoor cats. Further delays will amplify the problems. The sooner we take action, the better.

\section{Acknowledgments}

We thank S. Hess for fruitful discussions and T. Longcore and C. Rich for constructive suggestions on the draft manuscript.

\section{Literature Cited}

American Society for the Prevention of Cruelty to Animals (ASPCA). 2009. ASPCA estimates up to 1 million pets at risk during economic crisis. Press release. ASCPC, New York.

Blanton, J. D., C. A. Hanlon, and C. E. Rupprecht. 2007. Rabies surveillance in the United States during 2006. Journal of the American Veterinary Medical Association 231:540556.

Jessup, D. 2004. The welfare of feral cats and wildlife. Journal of the American Veterinary Medical Association 225:13771383.

Longcore, T., C. Rich, and L. M. Sullivan. 2009. Critical assessment of claims regarding management of feral cats by trap-neuter-return. Conservation Biology 23:887-894.

Nelson, M. P., and J. A. Vucetich. 2009. On advocacy by environmental scientists: what, whether, why, and how. Conservation Biology 23:1090-1101.

Schmidt, P. M., R. R. Lopez, and B. A. Collier. 2007. Survival, fecundity, and movements of free-roaming cats. Journal of Wildlife Management 71:915-919.

Winter, L. 2003. Popoki and Hawai'i's native birds. 'Elepaio 63:4346.

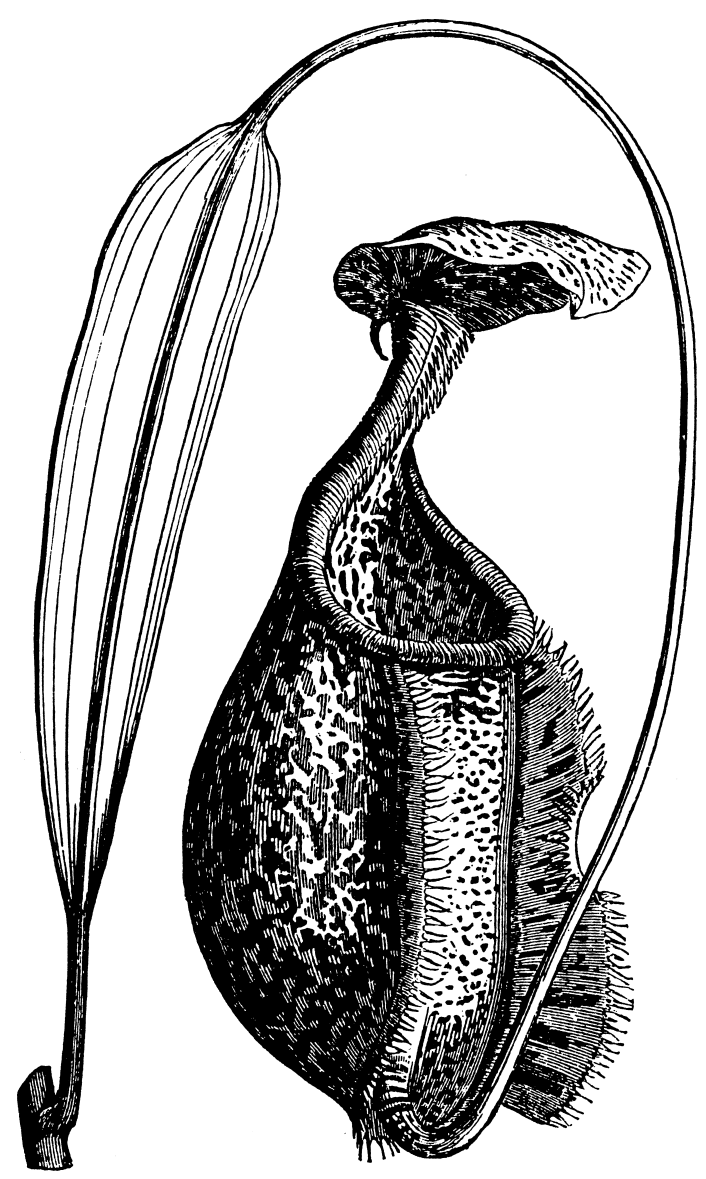

